\title{
Research on Operation Optimization Problem of Material Handling System for Large Ship
}

\author{
Cuiping Wen \\ Department of Control Science and \\ Technology \\ Huazhong University of Science \\ and Technology,Wuhan, China \\ Email:383305385@qq..com
}

\author{
Kai Huang \\ DeGroote School of Business \\ McMaster University, Hamilton, \\ Ontario L8S4M4, Canada \\ E-mail: khuang@mcmaster.ca
}

\author{
Weifeng Zhu* \\ Department of Control Science and \\ Technology \\ Huazhong University of Science \\ and Technology,Wuhan, China \\ Email: 123565899@qq.com
}

\begin{abstract}
Material supply is the basic guarantee for the large ship sailing to ocean. In order to improve the efficiency of the supply of the large ship, the support of materials handling system is needed. According to the typical material handling system of the large ship, with the material handling's time shortest as the decision goal and the handling sequence as the decision variable, the analysis and construction of the model for optimizing the system's operation is given in this paper. The improved genetic algorithm is put forward. The specific model is also given and it's proved that the analysis and modeling method in this paper can effectively improve the efficiency of the material handling of the large ships.
\end{abstract}

Keywords- Large ship; Material Handling System; Material Supply; Optimization.

\section{INTRODUCTION}

Large ship will constantly consume food, fuel, water and the task material when sailing in the ocean or being away from the security base for one mission. In order to guarantee the operation of the ship and the implementation of the mission, the material must be supplied rapidly. Large ship material handling system is the system that all material handling must be completed within the fixed time, according to the kinds and the quantities of the goods, combined with the structure of ship, with the inherent handling tools.

Material Handling System refers to a system or process which uses related equipments or devices to remove, store, protect and control material coordinately and reasonably. As a typical material handling system, the main problem of operation optimization problem of material handling system for large ship is to determine material handling solution which can accomplish material handing in relatively short time according to the specific supply task. The species, quantity and mutual constraint relations of materials are important factors to influence handling time. The system will generally cause certain queuing phenomenon.

There are few research literatures at home or abroad about queuing problem caused by material transportation which has few reference value either. Through the analysis, abstract and induction of the problem, we find it can be merged into the TSP problem which can be well solved. TSP is a classical NP-hard combinatorial optimization problem which has been widely researched by domestic and foreign scholars. An effective way to solve TSP is to put forward intelligent algorithms. However, all kinds of them have their advantages and disadvantages. And how to optimize them becomes a permanent research subject. Many domestic and foreign scholars solve the TSP problem through the study on the improved intelligent algorithm, YongJun Huang, etc ${ }^{[1]}$ and Vui Ann Shim, Kay Chen Tan, Chia Jun Yong ${ }^{[2]}$, in allusion to the traditional genetic algorithm, solve the limitation of the TSP problem, such as: precocious phenomena, local convergence is too fast and global "climbing" ability is not strong and so on, to solve the TSP problem through the improvement of genetic algorithm. And the improved algorithm has good feasibility and validity. According to the disadvantage of the ant colony algorithm in solving the TSP problem, such as: convergence slow, easy to fall into local optimum, Jin $\mathrm{Hao}^{[3]}$ and Gaifang Dong, William W. Guo, Kevin Tickle ${ }^{[4]}$ carry on the improvement. And the performance of the improved algorithm is significant improvement. According to the disadvantage of the simulated annealing algorithm in solving the TSP problems, such as, the whole grasp search ability is not enough, WeiBo Yang, YanWei Zhao ${ }^{[5]}$ and Shakouri, $\mathrm{GH}^{[6]}$ carry on the improvement, which can improve the performance and processes of the algorithm. According to the disadvantage of invalid solution and local optimum solution through the Hopfield neural network in solving the TSP problem, Ping $\mathrm{Li}^{[7]}$ carries on the improvement by combining the simulated annealing algorithm with Hopfield neural network. The algorithm has the advantage of fast convergence rate, avoiding invalid solution, being easy to access to the global optimal solution etc.

Based on the research and analysis of several typical intelligent algorithms, they are found that can be successfully applied to solve the TSP problem and each algorithm has its advantages and disadvantages. The appropriate algorithm can be chosen in view of the characteristics of the specific problem. Through the constant improvement and optimization, algorithm can reach the purpose of improving the performance. In allusion to the characteristics of the supplies sequence optimization in the large ship material handling system and the characteristic of each algorithm, the improved genetic algorithm can be designed to solve the problem. 


\section{ANALYSIS AND CONSTRUCTION OF THE MODEL OF OPERATION OPTIMIZATION PROBLEM}

The network structure of the typical material handling system for large ship is shown in the figure 1. And its process is as following:

1) The point $A$ is the boat for supply or the dock. The point $B$ is the working-storage section of the target ship. The point $C_{i}$ is the warehouse where the number $i$ material will be arrived.

2) There are $n$ kinds of materials that need to be transported from point $A$ to the warehouses $C_{1}, C_{2}, \ldots, C_{n}$.

3) The path from $A$ to $B$ can be used to transport next material after one material finishes the transportation from $A$ to $B$.

4) The material that arrives at $B$ can immediately transport to $C_{i}$ if there are not any other materials in relationship with it. Otherwise, it can't transport until the relative ones finish the transportation.

5) The material that arrives at $B$ obeys the rule of first come first service (FIFO)

6 ) The single time and the total time from $A$ to $B$ and $B$ to $C_{i}$ for each material are known.

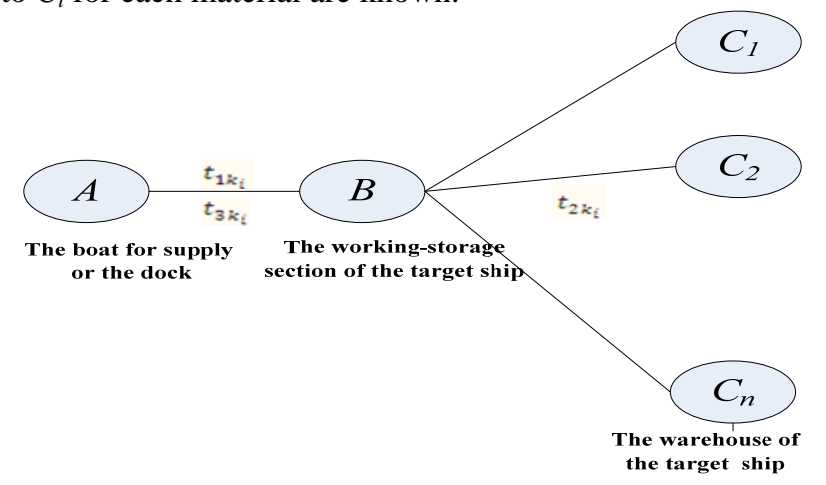

Figure 1. The network structure of the typical material handling system for large ship

When the handling order of materials from point $A$ to point $B$ was determined, the total time of the material's handling is established ${ }^{[8]}$. That is, the handling sequence from A to B is one-to-one relationship with the total time. Therefore, the model of operation optimization is with the material handling's time shortest as the decision goal and the handling order as the decision variable. The modeling process is as following:

\section{Parameters:}

A: The boat for supply or the dock;

$B$ : The working-storage section of the target ship;

$C_{i}$ : The warehouse that the $i$ material will to be arrived;

$k_{i}$ : The materials which is transported in the $i$ times, and the value can be $1,2, \ldots, n$;

$\mathrm{t}_{1 \mathrm{k}_{\mathrm{i}}}$ : The total time for $k_{i}$ transported from $A$ to $B$;

$\mathrm{t}_{2 \mathrm{k}_{\mathrm{i}}}$ : The total time for $k_{i}$ transported from $B$ to $C$;

$t_{3 k_{i}}$ : The single time for $k_{i}$ transported from $A$ to $B$;

$E_{k_{i}}$ : The moment for $k_{i}$ finished the transportation;

$\mathrm{S}_{\mathrm{k}_{\mathrm{i}}}$ : The moment for $k_{i}$ the first time to arrive at $B$;
$R_{k_{i} k_{j}}$ : If there is a mutual influence between material $k_{i}$ and material $k_{j}$, $=\left\{\begin{array}{l}0, \text { there is no influence between } k i \text { and } k j \\ 1, \text { there has influence between } k i \text { and } k j\end{array}\right.$;

$T_{k_{i} k_{j}}$ : The time that $k_{j}$ needs to wait for $k_{i}$ to complete transportation after arrives at $B$,

$$
=\left\{\begin{array}{ll}
E_{k_{i}}-S_{k_{j}}, & E_{k_{i}}-S_{k_{j}}>0 \\
0 & , E_{k_{i}}-S_{k_{j}} \leq 0
\end{array}\right. \text {; }
$$

$\left(k_{1}, k_{2}, \ldots, k_{n}\right)$ : The handling order of $\mathrm{n}$ kinds of materials, $\in H=\left\{\left(k_{1}, k_{2}, \ldots, k_{n}\right) \mid\left(k_{1}, k_{2}, \ldots, k_{n}\right)\right.$ is the arrangement of $1,2, \ldots, n\}$.

The objective function: The total time that all materials complete transportation gains to be shortest.

Constraint conditions: The path from $A$ to $B$ can only transport next kind of material after one finished. The materials which have influence with others can't in the path from $B$ to $C_{i}$ at the same time. The material that arrives at $B$ obeys the rule of FIFO.

For a given material handling order $\left(k_{1}, k_{2}, \ldots, k_{n}\right)$, the analysis process of handling time is as following.

1) The moment that all of $k_{1}$ arrive at the warehouse: $E_{k_{1}}=t_{3 k_{1}}+t_{2 k_{1}}$.

2) The first moment that $\mathrm{k} 2$ arrive at $B: S_{k_{2}}=t_{1 k_{1}}+t_{3 k_{2}}$. $R_{k_{1} k_{2}}$ is 1 if $k_{2}$ has influence with $k_{1}$. Otherwise, $R_{k_{1} k_{2}}$ is 0 . When $R_{k_{1} k_{2}}$ is 1 , we need to count the time $T_{k_{i} k_{j}}$ that $k_{2}$ needs to wait at $B$. And the moment that all of $k_{2}$ arrive at the warehouse: $E_{k_{2}}=t_{1 k_{1}}+t_{3 k_{2}}+t_{2 k_{2}}+R_{k_{1} k_{2}} * T_{k_{1} k_{2}}$.

3) The first moment that $k_{3}$ arrive at $B: S_{k_{3}}=t_{1 k_{1}}+$ $t_{1 k_{2}}+t_{3 k_{3}}$. The time $k_{3}$ needs to wait at $B: \max \left(R_{k_{1} k_{3}} *\right.$ $\left.T_{k_{1} k_{3}}, \quad R_{k_{2} k_{3}} * T_{k_{2} k_{3}}\right)$.

The moment that all of $k_{3}$ arrive at the warehouse: $E_{k_{3}}=t_{1 k_{1}}+t_{1 k_{2}}+t_{3 k_{3}}+t_{2 k_{3}}+\max \left(R_{k_{1} k_{3}} * T_{k_{1} k_{3}}, R_{k_{2} k_{3}}\right.$ $\left.* T_{k_{2} k_{3}}\right)$.

n) The first moment that $k_{n}$ arrive at $B: S_{k_{n}}=$ $\sum_{i=1}^{i=n-1} t_{1 k_{i}}+t_{3 k_{n}}$.The time $k_{n}$ needs to wait at $B$ : $\max \left(R_{k_{j} k_{n}} * T_{k_{j} k_{n}}\right), j=1,2, \ldots, \mathrm{n}-1$.

The moment that all of $k_{n}$ arrive at the warehouse:

$E_{k_{n}}=\sum_{i=1}^{i=n-1} t_{1 k_{i}}+t_{3 k_{n}}+t_{2 k_{n}}+\max \left(R_{k_{j} k_{n}} * T_{k_{j} k_{n}}\right)$, $j=1,2, \ldots, n-1$.

For a given material handling sequence, the biggest one in $E_{k_{i}}$ is the total time that all material complete transportation. For the full arrangement of all $n$ kinds of materials, search a sequence $\left(k_{1}, k_{2} \ldots k_{n}\right)$ to make the total time transportation minimum. That is the decision variables which is the handling sequence $\left(k_{1}, k_{2} \ldots k_{n}\right)$ from $A$ to $B$.

Objective function:

$T=\min \left(\max \left(t_{3 k_{1}}+t_{2 k_{1}}, \quad \sum_{i=1}^{i=t-1} t_{1 k_{i}}+t_{3 k_{t}}+t_{2 k_{t}}+\max \right.\right.$ $\left.\left.\left(R_{k_{j} k_{t}} * T_{k_{j} k_{t}}\right)\right)\right)$

Constraint conditions: 


$$
\left\{\begin{array}{c}
\left(k_{1}, k_{2}, \ldots, k_{n}\right) \in H=\left\{\left(k_{1}, k_{2}, \ldots, k_{n}\right) \mid\left(k_{1}, k_{2}, \ldots, k_{n}\right)\right. \\
\text { is the arrgement of } 1,2, \ldots, n\} \\
t=2,3, \ldots, n ; j=1,2, \ldots, t \\
R_{k_{j} k_{t}}=r\left[k_{j}-1\right]\left[k_{t}-1\right]
\end{array}\right.
$$

The sequence $\left(k_{1}, k_{2} \ldots k_{n}\right)$ corresponding the minimum of $T$ is the optimal handling order in the $n$ ! kind of the sequences.

\section{THE ALGORITHM AND SOLVING STEPS OF MODEL}

The improved genetic algorithm is used to get the optimal solution of this model. And the solving steps are as following:

(1)Coding: The solution of code path is used to change the solution's space of the $\mathrm{n}$ kind of material arrangement into bit string form as $\left(\mathrm{k}_{1}, \mathrm{k}_{2}, \ldots, \mathrm{k}_{\mathrm{n}}\right)$.

(2)Initialization: The generation of evolution is set 0 . The method of random initialization is used to initialize the population $p(0)$. The operator of genetic algorithms and parameters, including the selection operator, crossover operator, mutation operator, crossover rate $\left(p_{c}\right)$ and mutation rate $\left(p_{m}\right)$, the number of gene (the species of materials), the capacity of the group, the maximum genetic generation, etc. The structure array goods $[\mathrm{N}]$ is used to store the information of the $n$ kinds of material, including the number of materials (num), the total time from $A$ to $B(t 1)$, the total time from $B$ to $C(t 2)$, the single time from $A$ to $B(t 3)$. The relationship between the $n$ kinds of materials is shown by $r[\mathrm{~N}][\mathrm{N}]$. The structure of variable result is defined to store the results, including the order of the transportation (seq[N]), the finish time of all kinds of goods (time $[\mathrm{N}]$ ), the total time of material transport (totaltime).

(3)Define adaptive function: The total time of material transport that the handling sequence is $\left(\mathrm{k}_{1}, \mathrm{k}_{2}, \ldots, \mathrm{k}_{\mathrm{n}}\right)$ is defined as the cost of individual, which is inversely proportional with adaptive function. According to the sequence $\left(\mathrm{k}_{1}, \mathrm{k}_{2}, \ldots, \mathrm{k}_{\mathrm{n}}\right)$, which is stored into result.seq, the corresponding material transport time is calculated and stored into time $[\mathrm{N}]$. The biggest one in time[N] is the totaltime, that is individual cost.

(4)Each individual's cost in group $p(t)$ is calculated through the adaptive function.

(5)The next generation group $p(t+1)$ is formed from group $p(t)$ through the operation of selection, crossover and mutation as the following way:

a) The individual is chosen by the cost of individual with the method of sorting choice. And the individual with high degree has the higher probability to be retained. The lower one may be eliminated.

b) The new individuals which are put into $p(t)$ is interlaced by using exchange heuristic crossover operator ${ }^{[10]}$ according to the crossover probability $p_{c}$.

c) The new individuals in population is mutated by using inverted - insert hybrid mutation operator ${ }^{[11]}$ According to the mutation probability $p_{m}$ and increasing $p_{m}$ in the later evolution.

d) The new individuals is created after the operation of a, $\mathrm{b}$ and $\mathrm{c}$. and change $t$ to $t+1$.
(6) If the new group can meet the end condition (the optimal individual keeps 20 generation or $t$ achieves the maximum genetic generation), then the algorithm is over. And else, return to (4) to continue.

\section{EXAMPLE AND ANALYSIS OF MODEL}

It's supposed that there are 10 kinds of materials to be supply. It's known that the time is shown in table 1 and the

\begin{tabular}{|c|c|c|c|}
\hline \multicolumn{4}{|c|}{ TRANSPORTATION (MIN) } \\
\hline & $t_{1 k_{i}}$ & $t_{2 k_{i}}$ & $t_{3 k_{i}}$ \\
\hline Material 1 & 72 & 130 & 3 \\
\hline Material 2 & 65 & 144 & 5 \\
\hline Material 3 & 84 & 310 & 3 \\
\hline Material 4 & 42 & 165 & 2 \\
\hline Material 5 & 54 & 186 & 3 \\
\hline Material 6 & 74 & 235 & 2 \\
\hline Material 7 & 63 & 214 & 3 \\
\hline Material 8 & 45 & 189 & 3 \\
\hline Material 9 & 65 & 254 & 5 \\
\hline Material 10 & 42 & 156 & 2 \\
\hline
\end{tabular}
unit of time is min. The relationship between the 10 kinds of materials is showed by the two-dimensional array $r[10][10]$.

TABLE I. THE SCHEDULE OF MATERIAL

$$
r[10][10]=\left[\begin{array}{llllllllll}
0 & 1 & 0 & 0 & 1 & 0 & 0 & 0 & 1 & 0 \\
1 & 0 & 0 & 1 & 0 & 1 & 0 & 1 & 0 & 0 \\
0 & 0 & 0 & 0 & 1 & 0 & 1 & 0 & 1 & 1 \\
0 & 1 & 0 & 0 & 0 & 1 & 0 & 1 & 0 & 0 \\
1 & 0 & 1 & 0 & 0 & 0 & 1 & 0 & 0 & 0 \\
0 & 1 & 0 & 1 & 0 & 0 & 0 & 0 & 0 & 1 \\
0 & 0 & 1 & 0 & 1 & 0 & 0 & 0 & 1 & 0 \\
0 & 1 & 0 & 1 & 0 & 0 & 0 & 0 & 0 & 1 \\
1 & 0 & 1 & 0 & 0 & 0 & 1 & 0 & 0 & 0 \\
0 & 0 & 1 & 0 & 0 & 1 & 0 & 1 & 0 & 0
\end{array}\right]
$$

Modeling:

$T=\min \left(\max \left(t_{3 k_{1}}+t_{2 k_{1}}, \sum_{i=1}^{i=t-1} t_{1 k_{i}}+t_{3 k_{t}}+t_{2 k_{t}}+\max \right.\right.$ $\left.\left.\left(R_{k_{j} k_{t}} * T_{k_{j} k_{t}}\right)\right)\right)$

Constraint conditions:

$$
\left\{\begin{array}{c}
\left(k_{1}, k_{2}, \ldots, k_{10}\right) \in H=\left\{\left(k_{1}, k_{2}, \ldots, k_{10}\right) \mid\left(k_{1}, k_{2}, \ldots, k_{10}\right)\right. \\
\text { is the arrgement of } 1,2, \ldots, 10\} \\
t=2,3, \ldots, 10 ; j=1,2, \ldots, t \\
R_{k_{j} k_{t}}=\mathrm{r}\left[k_{j}-1\right]\left[k_{t}-1\right]
\end{array}\right.
$$

TABLE II. THE RESULTS OF MATERIAL HANDLING TIME

\begin{tabular}{|l|l|l|}
\hline \multicolumn{1}{|c|}{$\begin{array}{c}\text { Serial } \\
\text { Number }\end{array}$} & \multicolumn{1}{|c|}{ Handling Order } & Total Time (min) \\
\hline 1 & $(1,5,10,2,3,7,8,4,9,6)$ & 1097 \\
\hline 2 & $(2,6,5,3,1,9,8,4,10,7)$ & 1106 \\
\hline 3 & $(3,6,10,2,1,9,8,4,7,5)$ & 1249 \\
\hline 4 & $(5,6,2,3,1,9,7,4,10,8)$ & 1033 \\
\hline 5 & $(6,8,9,5,3,4,2,1,10,7)$ & 902 \\
\hline
\end{tabular}




\begin{tabular}{|l|l|l|}
\hline 6 & $(7,5,9,8,3,1,2,4,10,6)$ & 1172 \\
\hline 7 & $(9,8,6,4,3,5,2,7,10,1)$ & 969 \\
\hline 8 & $(3,2,1,4,7,5,10,8,9,6)$ & 781 (Optimum) \\
\hline
\end{tabular}

Through the improved genetic algorithm, the optimal solution is searched with 0.031 seconds when proceeded to 4th generation. Part of the calculation results is shown in the table II. When the material type $n$ is large, the improved genetic algorithm is an ideal choice to solve this problem in the quality, speed and performance of getting the solution quality. The material handling order solved is applied to the actual ship material supply system, guiding the operation of the system. The results of the actual operation can be concluded that the theoretical result is matched with the actual result. And it's proved that the modeling and the solution are effective.

\section{CONCLUSION}

The construction of the model for optimizing the system's operation is given, with the handling order as the decision variable, according to the typical material handling system of the large ship in this paper. The solution of this model can be merged into the TSP problem. The improved genetic algorithm is successfully adopted to solve this model. Finally, it's proved that the modeling and the solution of this model are effective through the modeling, solving and the contrast to the actual operation situation according to the practical example of the model.

\section{ACKNOWLEDGMENT}

This work was supported by 701 research institute's project "Research on Material Handling System for Large Ship”.

\section{REFERENCES}

[1] YongJun Huang, Youxin Wu, and Huabin Liu, The Improved Genetic Algorithm is Presented to Solve the TSP Problem[J], Computer Engineering and Design, 2007, 28(24):5909-5911.

[2] Vui Ann Shim, Kay Chen Tan, and Chia Jun Yong, Evolutionary Algorithms for Solving Multi-objective Travelling Salesman Problem[J], FLEXIBLE SERVICES AND MANUFACTURING JOURNAL, 2011, 23(2):207-241.

[3] Jin Hao, Libao Shi, and JIaqi Zhou, The Random Disturbance Ant Colony Algorithm to Solve the Complex TSP Problem[J], System Engineering Theory and Practice, 2002, 22(9):88-91.

[4] Gaifang Dong, William W. Guo, and Kevin Tickle, Solving the Traveling Salesman Problem Using Cooperative Genetic Ant Systems[J], Expert System with Applications, 2012,39(5):5006 -5011.

[5] Weibo Yang and Yanwei Zhao, The Improved Simulated Annealing Algorithm to Solve the TSP Problem[J], Computer Engineering and Application,2010,46(15):34-36.

[6] Shakouri. GH, Shojaee. K, and Behnam. TM, Investigation on the Choice of the Temperature in the Simulation Annealing: A Mushy State SA for TSP[C], 17th Mediterranean Conference on Control and Automation, Thessaloniki, GREECE, 2009(1-3):1050-1055.

[7] Ping Li, Leifu Gao, and Xuwang Liu, An Algorithm Based on Simulated Annealing and Hopfield neural Network to Solve the TSP Problem[J], Science and Technology and Engineering, 2008, 8(14):3937-3939.
[8] S. M. Johnson, Optimal Two and Three -stage Production Schedules With Setup Times Included[J], Naval Research Logistics Quarterly, 1954, 1(1):61-68. 